\title{
Reproduction in premature ovarian insufficiency patients - from latest studies to therapeutic approach
}

\author{
Błażej Męczekalski, Marzena Maciejewska-Jeske, Agnieszka Podfigurna \\ Department of Gynaecological Endocrinology, Poznan University of Medical Sciences, Poznan, Poland
}

\begin{abstract}
Normal function of the ovaries, which is responsible for the hormonal and reproductive processes, is one of the most important determinants of fertility. Premature ovarian insufficiency (POI) is defined as cessation of menstrual cycle, increased serum follicle-stimulating hormone (FSH) levels, and decrease serum oestradiol levels in women before the age of 40 years. POI concerns about $1 \%$ of women and is characterised by severely diminished fertility. For the POI patient, this is one of the most dramatic problems. It influences their psychological status and functioning in society. The chance for spontaneous conception is very limited and ranges from 4 to $8 \%$. For contemporary medicine, infertility treatment in $\mathrm{POI}$ patients is a challenge. The problem is that there are no effective therapies to augment ovarian activity in $\mathrm{POI}$ patients. At present, oocyte donation is regarded as the only proven method in the treatment of infertility in POI patients. However, nowadays we can observe important progress in the development of fertility preservation methods. In the POI field it refers to cryopreservation of oocytes, embryos, and ovarian tissue. Additionally, new methods known as in vitro activation of dormant follicles and possible use of stem cells should be mentioned.
\end{abstract}

Key words: premature ovarian failure, oocyte donation, fertility, fertility preservation, in vitro maturation.

\section{Introduction}

Oogenesis and folliculogenesis are very complex, not fully understood processes. Research on oogenesis has an important impact on the study of female fertility. William Harvey, almost 400 years ago, wrote: Ex ovo omnia which in English means "all that is alive comes from the egg". In humans oogenesis starts quite early because three weeks after fertilisation primordial germ cells (PGCs) arise from the yolk sac and migrate through the hindgut to the genital ridge [1]. During this migration process rapid mitotic divisions take place. Maximal number of oocytes (6-8 million) occurs in mid-gestation at around the $20^{\text {th }}-24^{\text {th }}$ week of gestation. Follicle formation occurs around the $16^{\text {th }}$ $18^{\text {th }}$ week of gestation. Later a permanent decrease in the oocyte pool is observed. At birth the pool of oocytes is around 1 million. At the time of puberty the oocyte pool reaches 0.5 million. Close to the menopause the oocyte pool is very limited at only a few thousand [2].

\section{Premature ovarian insufficiency}

Premature ovarian insufficiency (POI) is defined as cessation of menstrual cycle, increased serum follicle-stimulating hormone (FSH) levels, and decreased serum oestradiol levels in women before the age 40 years [3].
POI concerns about $1 \%$ of women and is characterised by severely diminished fertility. The aetiopathogenesis of this disorder is related to a multifactoral background. A major part is regarded as idiopathic, although it is suspected to be genetically determined. An important part of POI aetiopathogenesis is related to known genetic background $[4,5]$. Autoimmunological causes are involved in the pathogenesis of $4-30 \%$ of POI cases [6]. The presence of antiovarian autoantibodies (AOAs), lymphocytic oophoritis, and associated autoimmune disorders are key evidence of autoimmunological background. There is POI association with other autoimmune diseases. Hypothyroidism is the most common associated autoimmune disorder with POI. Diabetes mellitus occurs together with POI in $2.5 \%$ of cases. Approximately $10-20 \%$ of patients with Addison disease have POI. The increasing number of POI cases is also related to iatrogenic background (use of radiotherapy, chemotherapy, and pelvic surgery in patients treated due to oncological diseases). The described oncological treatment has an important impact on fertility in women [7].

\section{Spontaneous fertility in POI}

Spontaneous fertility in POI patients is dramatically limited [8]. Patients are characterised by oligomenor- 
rhoea and amenorrhoea. Although up to $25 \%$ of patients can ovulate, only $5 \%$ to $10 \%$ will conceive and deliver after being diagnosed with POI [9]. This study was conducted on quite a large number $(N=358)$ of patients with POI.

For contemporary medicine, infertility treatment in $\mathrm{POI}$ patients is a real challenge. The problem is that there are no effective therapies to augment ovarian activity in POI patients. At present, oocyte donation is regarded as the only proven method in the treatment of infertility in POI patients. Another study from 1995 presented lower $(2.5 \%)$ chance for conception in $\mathrm{POI}$ patients. Generally, it is important to advise POI patients not to postpone plans to be pregnant.

\section{Oocyte donation}

Oocyte donation has quite a long history; the first oocyte donation was performed in 1984 [10]. The number of such procedures in the US and Europe is increasing. POI is the main indication to use oocyte donation in $\mathrm{POI}$ women who desire to be pregnant. Oocyte donation in $\mathrm{POI}$ patients presents quite high efficacy. According to Ameratunga et al. [11], the pregnancy rate after an oocyte donation cycle is around $40 \%$, and cumulative pregnancy rates after four cycles reach $70-80 \%$.

Turner syndrome patients are also candidates for in vitro fertilisation with oocyte donation. However, it is well known that in these patients a high rate of cardiovascular anomalies is observed. Therefore, according to the American Society of Reproductive Medicine (ASRM), women with Turner syndrome before attempting to become pregnant should undergo medical and cardiovascular control [12]; this can decrease the high rate of cardiovascular mortality during pregnancy. The procedure of oocyte donation can be used also in cancer survivors.

Recently, numerous studies evaluated the impact of oocyte donation on maternal and foetal outcomes. Oocyte donation pregnancies are associated with a higher rate of placental disorders of pregnancy, such as gestational hypertension and pre-eclampsia [13].

\section{Fertility preservation}

Advancement in fertility preservation gives an optimistic perspective for many POI patients. Gonadal function of women who will undergo radiotherapy (for oncological reasons) can be protected by surgical transposition of ovaries out of the pelvis [14]. Some experimental methods can be performed in women who anticipate undergoing chemotherapy with gonadotoxic agents. It is recommended that gonadotropin-releasing hormone analogues be used before chemotherapy to suppress ovarian function [15]. However, there are conflicting data regarding the efficacy of this therapy.
According to the American Society of Clinical Oncology (ASCO) from 2013, there is insufficient evidence concerning the effectiveness of ovarian suppression with $\mathrm{GnRH}$ analogues as a fertility preservation method.

Fertility preservation can concern cryopreservation of oocytes, embryos, and ovarian tissue. There are still technical problems with the cryopreservation of oocytes because oocytes contain more water and are more susceptible to cryoinjury.

Cryopreservation of ovarian tissue can also be a method for women who have hormone-sensitive malignancies, and for women anticipating haematopoietic stem cell transplantation for the treatment of benign haematological diseases (sickle cell anaemia, thalassemia major, aplastic anaemia) [16].

Transplantation of ovarian tissue into a pelvic site is named orthotopic, and into an extrapelvic site (abdominal wall or forearm) is named heterotopic. So far, pregnancies and live births have been revealed only with orthotopic transplantation of cortical tissue.

In 2004 livebirth occurred after orthotopic autotransplantation of cryopreserved tissue from women diagnosed previously with stage IV Hodgkin lymphoma [17].

Ovarian tissue cryopreservation and transplantation is still regarded as experimental. The biggest worldwide report on ovarian transplantation tissue was published in 2016. It includes 95 orthotopic transplantations of ovarian tissue in 74 women after cytotoxic treatment in a fertility preservation network (16 centres in Europe). Twenty-one pregnancies and 17 deliveries were reported [18].

\section{Characterisation of the new method - in vitro activation}

Recently, important progress has been observed in the field of a new method related to infertility treatment in POI patients. This new treatment was named as in vitro activation (IVA) of dormant follicles [19]. Residual dormant follicles of POI patients' follicles are difficult to grow spontaneously, and thus the patients are unlikely conceive with their own oocytes. A number of intraovarian factors have been shown to be important for primordial follicle activation. IVA implements ovarian fragmentation disruption of Hippo signalling pathway and treatment with phosphatidylinositol-3kinase (PI3K) stimulator to activate dormant primordial and restrained secondary and preantral follicles in POI patients. A full cycle of IVA includes laparoscopic surgery to remove the ovary, which is subsequently cut into cortical strips and vitrified. After thawing of cryopreserved ovarian tissues, the ovarian strips are further fragmented and incubated for two days with PI3K stimulators. After that, the ovarian strips are autografted under laparoscopic surgery. Then the patient undergoes full protocol of ovarian stimulation and IVF procedure. 
Currently, two healthy babies were delivered, together with two additional pregnancies. The inventors of the method admit that to improve the efficiency of IVA it is important to develop a noninvasive method to predict the presence of residual follicles before the first laparoscopy [20]. Controlled studies would be required before IVA can be advocated for more widespread clinical use.

\section{Possible use of stem cells}

Stem cells have self-renewal and regeneration potential; hence they can be very effective in the treatment of ovarian failure and consequently infertility [21]. There are several kinds of stem cells, such as: mesenchymal stem cells (MSCs), stem cells from extraembryonic tissues, induced pluripotent stem cells (iPSCs), and ovarian stem cells that are used in POI stem cell therapy, as observed in previous studies.

Stimpfel et al. [22] successfully characterised and differentiated in vitro stem cells from the adult human ovarian cortex. The question is related to the application of these cells into the therapy of infertility in POI patients. It may open a completely new chapter in the treatment of premature ovarian insufficiency.

\section{Conclusions}

POI patients suffer from short- and long-term consequences. Infertility issues are the most dramatic problems for POI women. They have also an important, negative impact on psychological status and quality of life. Proper diagnosis and treatment of infertility has fundamental significance. Nowadays new methods of infertility treatment in POI patients are being developed.

\section{Disclosure}

The authors report no conflict of interest.

\section{References}

1. MacLennan M, Crichton JH, Playfoot CJ, Adams IR. Oocyte development, meiosis and aneuploidy. Semin Cell Dev Biol 2015; 45: 68-76.

2. Wang JJ, Ge W, Liu JC, et al. Complete in vitro oogenesis: retrospects and prospects. Cell Death Differ 2017; 24: 1845-1852.

3. The ESHRE Guideline Group on POI, Weber L, Davies M, Andersdon R, et al. ESHRE Guideline: management of women with premature ovarian insufficiency. Hum Reprod 2016; 31: 926-937.

4. Meczekalski B, Podfigurna-Stopa A. Genetics of premature ovarian failure. Minerva Endocrinol 2010; 35: 195-209.

5. Fenton AJ. Premature ovarian insufficiency: Pathogenesis and management. J Midlife Health 2015; 6: 147-153.

6. Bakalov VK, Anasti JN, Calis KA, et al. Autoimmune oophoritis as a mechanism of follicular dysfunction in women with $46, \mathrm{XX}$ spontaneous premature ovarian failure. Fertil Steril 2005; 84: 958-965.

7. Podfigurna-Stopa A, Czyzyk A, Grymowicz M, et al. Premature ovarian insufficiency: the context of long-term effects. J Endocrinol Invest 2016; 39: 983-990.
8. Rebar RW. Premature ovarian failure. Obstet Gynecol 2009; 113: 13551363.

9. Bidet $M$, Bachelot $A$, Bissauge $E$, et al. Resumption of ovarian function and pregnancies in 358 patients with premature ovarian failure. J Clin Endocrinol Metab 2011; 96: 3864-3872.

10. Lutjen P, Trounson A, Leeton J, et al. The establishment and maintenance of pregnancy using in vitro fertilization and embryo donation in a patient with primary ovarian failure. Nature 1984; 307: 174.

11. Ameratunga D, Weston G, Osianlis T, et al. In vitro fertilisation (IVF) with donor eggs in post-menopausal women: are there differences in pregnancy outcomes in women with premature ovarian failure (POF) compared with women with physiological age-related menopause? J Assist Reprod Genet 2009; 26: 511-514.

12. Practice Committee of American Society For Reproductive Medicine. Increased maternal cardiovascular mortality associated with pregnancy in women with Turner syndrome. Fertil Steril 2012; 97: 282.

13. Savasi VM, Mandia L, Laoreti A, Cetin I. Maternal and fetal outcomes in oocyte donation pregnancies. Hum Reprod Update 2016; 22: 620-633.

14. Oktay K, Harvey BE, Loren AW. Fertility Preservation in Patients With Cancer: ASCO Clinical Practice Guideline Update Summary. J Oncol Pract 2018; 14: 381-385.

15. Ethics Committee of American Society for Reproductive Medicine. Fertility preservation and reproduction in patients facing gonadotoxic therapies: a committee opinion. Fertil Steril 2013; 100: 1224-1231.

16. Albani E, Bracone G, Biccari SD, et al. Human ovarian tissue cryopreservation as fertility reserve. In: Topics in cancer survivorship. Mohan R (ed.). IntechOpen Limited, London 2012: 215.

17. Donnez J, Dolmans MM, Demylle D, et al. Live birth after orthotopic transplantation of cryopreserved ovarian tissue. Lancet 2004; 364: 1405-1410.

18. Van der Ven H, Liebenthron J, Beckmann M, et al. Ninety-five orthotopic transplantations in 74 women of ovarian tissue after cytotoxic treatment in a fertility preservation network: tissue activity, pregnancy and delivery rates. Hum Reprod 2016; 31: 2031-2041.

19. Zhai J, Yao G, Dong F, et al. In Vitro Activation of Follicles and Fresh Tissue Auto-transplantation in Primary Ovarian Insufficiency Patients. J Clin Endocrinol Metab 2016; 101: 4405-4412.

20. Kawashima I, Kawamura K. Regulation of follicle growth through hormonal factors and mechanical cues mediated by Hippo signaling pathway. Syst Biol Reprod Med 2018; 64: 3-11.

21. Sheikhansari G, Aghebati-Maleki L, Nouri M, et al. Current approaches for the treatment of premature ovarian failure with stem cell therapy. Biomed Pharmacother 2018; 102: 254-262.

22. Stimpfel M, Skutella T, Cvjeticanin B, et al. Isolation, characterization and differentiation of cells expressing pluripotent/multipotent markers from adult human ovaries. Cell Tissue Res 2013; 354: 593-607. 